\title{
The RadioAstron Dedicated DiFX Distribution
}

\author{
Gabriele Bruni ${ }^{1, *}$, James M. Anderson ${ }^{2}$, Walter Alef ${ }^{1}$, Helge Rottmann ${ }^{1}$, Andrei P. Lobanov ${ }^{1,3}$ \\ and J. Anton Zensus ${ }^{1}$ \\ 1 Max-Planck-Institut für Radioastronomie, Auf dem Hügel 69, 53121 Bonn, Germany; \\ alef@mpifr-bonn.mpg.de (W.A.); hrottmann@mpifr-bonn.mpg.de (H.R.); \\ alobanov@mpifr-bonn.mpg.de (A.P.L.); azensus@mpifr.de (J.A.Z.) \\ 2 Deutsches GeoForschungsZentrum GFZ, Telegrafenberg A6, 14473 Potsdam, Germany; \\ anderson@gfz-potsdam.de \\ 3 Institut für Experimentalphysik, Universität Hamburg, Luruper Chaussee 149, 22761 Hamburg, Germany \\ * Correspondence: bruni@mpifr-bonn.mpg.de; Tel.: +49-228-525-292
}

Academic Editors: Jose L. Gómez, Alan P. Marscher and Svetlana G. Jorstad

Received: 18 July 2016; Accepted: 17 October 2016; Published: 27 October 2016

\begin{abstract}
Distributed FX-architecture (DiFX) is a software Very Long Baseline Interferometry (VLBI) correlator currently adopted by several main correlation sites around the globe. After the launch of the RadioAstron Space-VLBI mission in 2011, an extension was necessary to handle processing of an orbiting antenna, to be correlated with supporting ground arrays. Here, we present a branch of the main DiFX distribution (2.4), uploaded on the publicly available repository during July 2016, that the Max Planck Institute for Radio Astronomy (MPIfR) developed to process data of the three key active galactic nuclei (AGN)-imaging RadioAstron science projects, as well as part of the AGN survey project, and General Observing Time (GOT) projects proposed since Announcement of Opportunity 2 (AO-2, July 2014-July 2015). It can account for general relativistic correction of an orbiting antenna with variable position/velocity, providing a routine to convert the native RadioAstron Data Format (RDF) format to the more common Mark5 B (M5B). The possibility of introducing a polynomial clock allows one to mitigate the effects of spacecraft acceleration terms in near-perigee observations. Additionally, since for the first time polarimetry on space-baselines is available thanks to RadioAstron, this DiFX branch allows one to include the spacecraft orientation information at the correlation stage, in order to perform proper polarization calibration during data reduction. Finally, a fringe-finding algorithm able to manage an arbitrarily large fringe-search window is included, allowing one to increase the search space normally adopted by common software packages like HOPS.
\end{abstract}

Keywords: VLBI; space-VLBI; correlation; RadioAstron; AGN

\section{Introduction}

The Distributed FX-architecture (DiFX) software correlator was first introduced in 2007 [1] to offer a versatile alternative to the previous generation of hardware correlators. Designed to run on computer clusters, it was initially adopted by Swinburne University (Australia) for the Long Baseline Array (LBA) correlation. Subsequently, it was also installed at the National Radio Astronomy Observatory (NRAO, USA), as well as by single users. Indeed, due to the exponential improvement of computing facilities, DiFX can now be run also on a single powerful machine, given a modest volume of input data. In 2011, a second version was made available to the community (DiFX-2, [2]), including a number of improvements and new utilities, including multiple phase-center correlation in a single pass, phase-calibration tones extraction for geodetic applications, zoom-mode for correlation of disparate but overlapping sub-bands, full support for VDIF data format, arbitrary-order polynomial clock, and many more. 
The RadioAstron space radio telescope was launched in July 2011 by the Russian space agency (Roscosmos) and it is led by Astro Space Center (ASC, Moscow). It is a 10-m single dish, mounted onboard the Spekt- $R$ satellite, and is able to perform interferometry observations with ground arrays of radio telescopes [3]. It can reach extreme baselines of $\sim 25$ Earth diameters, thanks to an elliptical orbit with an apogee of $\sim 330.000 \mathrm{~km}$-almost the distance between Earth and the Moon. For the first time since the VSOP mission in the 1990s, a space-VLBI mission required correlation with ground antennas, thus implying a number of complications given by an orbiting (moving) telescope, under the influence of both Earth and Moon gravitational fields. As a contribution to the mission, the Max Planck Institute for Radio Astronomy (MPIfR) developed a dedicated version of the DiFX, named ra-DiFX, implementing the code necessary to deal with RadioAstron [4]. It is now running on the MPIfR cluster, and is used to process data from three Key Science Projects (KSPs) aiming at imaging AGN jets at the highest angular resolution possible from space. With this paper, we present the second, improved version of this publicly-available software, branched from DiFX 2.4 in September 2015.

\section{The RadioAstron DiFX Distribution}

As described in [4], the ra-DiFX implements, since the first version, additional code that allows the handling of an antenna with an arbitrary position and velocity in time, but still these modifications do not involve the main algorithm used for data correlation of ground antennas by DiFX. Indeed, mainly the delay model server, Calc, from the Calc/Solve package (http:/ / gemini.gsfc.nasa. gov/solve/) was modified in order to take into account the orbit and general relativistic corrections due to Earth and Moon gravitational fields. Additionally, the DiFX metadata system was modified to deal with the changing position and velocity of the spacecraft as a function of time.

In the following, a list of the modifications and capabilities already present in the first branch is given:

- Implementing RadioAstron Data Format (RDF)-Mark5B conversion routine, to read in data from the RadioAstron spaceborne antenna

- Enabling delay model server Calc (Calc/Solve Package) to calculate delay information for a spaceborne antenna

- Introducing general relativistic corrections in the delay model

- Changing the DiFX metadata system to deal with variable position/velocity of the spaceborne antenna

- Calculating the delay for the transmission of the signal from the spacecraft to the tracking station

- Calculating the equivalent of parallactic angle correction for the spaceborne antenna from the antenna orientation obtained from the telemetry information, particularly useful for polarization calibration.

- Enabling the use of wide fringe-search windows, thanks to the customized fringe-fitting software.

Consistency checks with the ASC correlator ([3]) have been performed at an initial stage (2013), by means of the correlation of a number of experiments with both pieces of software and comparing results. These have shown to be compatible within errors [5]. The second ra-DiFX version presented here is based on the same space-VLBI code extension, and thus its validity is still verified.

The main motivation for the production of a second branch was to include all of the DiFX enhancements introduced since 2010, thus improving the efficiency of the KSPs' data processing. Full VDIF compatibility is now available, and moreover, the possibility of introducing a polynomial clock allows for mitigation of the residual fringe rate due to acceleration terms of the spacecraft, especially important for near-perigee observations like the AGN-imaging projects. In Figure 1, an example of this effect is visible from AIPS plots as a steepening of the rate solutions towards the beginning of the experiment, where RadioAstron approaches the perigee (AO-1 observations of BL Lac from the AGN Polarization KSP, [6]). While the applied calibration in AIPS could compensate for this effect, a strong signal-to-noise ratio (SNR) is necessary to obtain solutions close enough in time to 
properly fit the trend. This might not be the case for faint sources, for which baseline stacking in the data-reduction stage is needed, resulting in a loss of signal. Thus, the polynomial clock feature of this new version of $r a$-DiFX can help in recovering signal by properly setting the correlation window across the experiment, following the spacecraft delay/rate trend in time. As for the previous branch, the possibility of including the spacecraft orientation information at the correlation stage allows one to properly calibrate polarization with standard data reduction packages. Indeed, the polarimetry capabilities on space-baselines - offered for the first time by RadioAstron - have already demonstrated to be successful for the AGN Polarization KSP, showing stable D-terms of a few percent for the spacecraft [6,7].

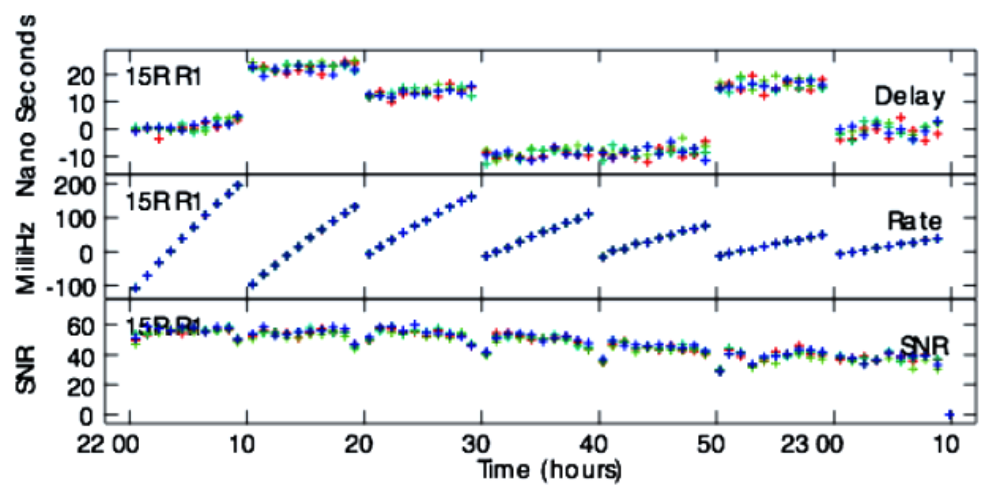

Figure 1. Example of residual acceleration terms for RadioAstron, visible as a steepening of the fringe-rate solutions near to perigee (towards left side of the timeline).

The ra-DiFX software correlator is regularly used at the MPIfR to correlate RadioAstron KSP and General Observing Time (GOT) projects. Since July 2016, it has also been publicly available on the DiFX repository (https://svn.atnf.csiro.au/difx/master_tags/DiFX-RA-1.0.0) for download and use under the sGPL license.

Acknowledgments: The RadioAstron project is led by the Astro Space Center of the Lebedev Physical Institute of the Russian Academy of Sciences and the Lavochkin Scientific and Production Association under a contract with the Russian Federal Space Agency, in collaboration with partner organizations in Russia and other Countries. This research is based on observations correlated at the Bonn Correlator, jointly operated by the Max-Planck-Institut für Radioastronomie (MPIfR), and the Federal Agency for Cartography and Geodesy (BKG).

Conflicts of Interest: The authors declare no conflict of interest.

\section{Abbreviations}

The following abbreviations are used in this manuscript:

$\begin{array}{ll}\text { AGN } & \text { Active Galactic Nuclei } \\ \text { AO } & \text { Announcement of Opportunity } \\ \text { ASC } & \text { Astro Space Center } \\ \text { DiFX } & \text { Distributed FX } \\ \text { GOT } & \text { General Observing Time } \\ \text { KSP } & \text { Key Science Project } \\ \text { M5B } & \text { Mark5 B } \\ \text { MDPI } & \text { Multidisciplinary Digital Publishing Institute } \\ \text { MPIfR } & \text { Max-Planck Institut für Radioastronomie } \\ \text { RDF } & \text { RadioAstron Data Format } \\ \text { VLBI } & \text { Very Long Baseline Interferometry }\end{array}$




\section{References}

1. Deller, A.T.; Tingay, S.J.; Bailes, M.; West, C. DiFX: A software correlator for very long baseline interferometry using multiprocessor computing environments. Publ. Astron. Soc. Pac. 2007, 119, 318-336.

2. Deller, A.T.; Brisken, W.F.; Phillips, C.J.; Morgan, J.; Alef, W.; Cappallo, R.; Middelberg, E.; Romney, J.; Rottmann, H.; Tingay, S.J.; et al. DiFX-2: A more flexible, efficient, robust, and powerful software correlator. Publ. Astron. Soc. Pac. 2011, 123, 275-287.

3. Kardashev, N.S.; Khartov, V.V., Abramov, V.V.; Avdeev, V.Y.; Alakoz, A.V.; Aleksandrov, Y.A.; Ananthakrishnan, S.; Andreyanov, V.V.; Andrianov, A.S.; Antonov, N.M.; et al. "RadioAstron"-A telescope with a size of 300,000 km: Main parameters and first observational results. Astron. Rep. 2013, 57, 153-194.

4. Bruni, G.; Anderson, J.M.; Alef, W.; Lobanov, A.; Zensus, J.A. Space-VLBI with RadioAstron: New correlator capabilities at MPIfR. In Proceeding of the 12th European VLBI Network Symposium and Users Meeting, Cagliari, Italy, 7-10 October 2014.

5. Kovalev, Y.Y.; Kardashev, N.S.; Kellermann, K.I.; Lobanov, A.P.; Johnson, M.D.; Gurvits, L.I.; Voitsik, P.A.; Zensus, J.A.; Anderson, J.M.; Bach, U.; et al. RadioAstron Observations of the Quasar 3C273: A Challenge to the Brightness Temperature Limit. Astrophys. J. Lett. 2016, 820, L9.

6. Gómez, J.L.; Lobanov, A.P.; Bruni, G.; Kovalev, Y.Y.; Marscher, A.P.; Jorstad, S.G.; Mizuno, Y.; Bach, U.; Sokolovsky, K.V.; Anderson, J.M.; et al. Probing the innermost regions of AGN jets and their magnetic fields with RadioAstron. I. imaging BL Lacertae at $21 \mu$ as resolution. Astrophys. J. 2016, 817, 96-109.

7. Lobanov, A.P.; Gómez, J.L.; Bruni, G.; Kovalev, Y.Y.; Anderson, J.; Bach, U.; Kraus, A.; Zensus, J.A.; Lisakov, M.M.; Sokolovsky, K.V.; et al. RadioAstron space VLBI imaging of polarized radio emission in the high-redshift quasar $0642+449$ at $1.6 \mathrm{GHz}$. Astron. Astrophys. 2015, 583, A100.

(C) 2016 by the authors; licensee MDPI, Basel, Switzerland. This article is an open access article distributed under the terms and conditions of the Creative Commons Attribution (CC-BY) license (http://creativecommons.org/licenses/by/4.0/). 